\title{
A Wireless Sink Node Using Information Fusion for Water Quality Information Collection in Factory Aquaculture
}

\author{
Yuting Yang, Haijiang Tai, Daoliang $\mathrm{Li}^{*}$, and Yaoguang Wei \\ College of Information and Electrical Engineering, China Agricultural University, \\ Beijing 100083, P.R. China \\ dliangl@cau.edu.cn
}

\begin{abstract}
Water quality information collection is an important part of factory aquaculture. This paper proposes a kind of wireless sink nodes using information fusion for water quality information collection in factory aquaculture. In the sink nodes, Support Vector Regression and fuzzy algorithmic approach are used for information fusion. Making decisions according to information fusion, it converts the collected water quality information into a simple parameter that signified the current state of water quality. The sink nodes can eliminate redundant information, reduce information transmission, thus save energy effectively and prolong the network life.
\end{abstract}

Keywords: the wireless sink node, information fusion, Support Vector Regression, Fuzzy Algorithmic Approach.

\section{Introduction}

In china, the traditional aquaculture is usually in the consumption of natural resources and at the cost of environmental pollution. Recently, the factory aquaculture has occupied an important position in china, and been the representative of the advanced agriculture and the trend of agricultural modernization. So the development of factory aquaculture is the inevitable requirement to realize the fishery breeding sustainable development. As we know, infrastructure equipment modernization is the basic condition to develop factory farming. Similarly, as the important component of factory aquaculture, water quality monitoring is the key link to promote factory aquaculture toward automation, large-scale, high yield and high quality. In monitoring area, sensor nodes usually gather all kinds of water quality information, such as temperature, light, dissolved oxygen, $\mathrm{Ph}$, etc, which codetermine to the current water quality conditions[1-2].

Presently, the great majority water quality monitoring systems adopt the same method that data which was collected by each node will be transferred to the monitoring center directly, besides the monitoring center process data and send the

\footnotetext{
Corresponding author.
} 
control instruction. But this method brings so big burden that transmission speed is slow. Moreover, many of the data transmission are redundant information for the monitoring center. Actually, the monitoring center just pays attention to the abnormal water quality conditions, and it will take control measures about water environment in abnormal conditions [3-5].

This paper, we refer to add the wireless sink node gather into the water quality monitoring system. Through information fusion at the sink node, all kinds of complicated water quality information will be converted into a simple parameter, and only send the abnormal water environmental data, which can extremely eliminate redundant information, effectively reduce the input data, thus save energy, prolong the network life, and improve the reaction of the whole monitoring system validly and accurately.

\section{Hardware Structure Design}

Figure 1 is the structure diagram of the wireless sink node, the device works as follows: As the CPU of the wireless sink node, the chip S3C2410 that was made by Samsung Company integrated the ARM920T core processor with 32-bits micro controller. The microprocessor contains $16 \mathrm{~KB}$ instructions, 16KB data cache, LCD controller, RAM controller, NAND flash controller, a $12 \mathrm{C}$ bus controller, a $12 \mathrm{~S}$ bus controller, four PWM timer and an internal timer, touch screen interface, two USB interface controller, four DMA channel, three UART, two SPI, parallel I/O interface, and maximum basic frequency is $203 \mathrm{MHz}$. Depending on the rich peripherals and interface, it can accomplish some full-featured application under the condition of low cost and low power consumption. SPI serial port makes it possible to connect S3C2410 with XBEE chips, which realizes the data transmission between the embedded platform and the sensor nodes via ZigBee network; Two-way UART serial port connect respectively with MC35I chip and MAXI490 chip, so as to realize cable connection between the sink node and the monitoring centers, then accomplish data and control instructions transmission and related data storage via the GPRS network [6].

This equipment introduces dual mode communication. On the one hand, data which are collected by each sensor node that is deployed in the factory aquaculture area will be transferred to the wireless sink node through ZigBee communication module based on the RF chip XBEE. Furthermore, the on-site environment parameters and control instructions are sent via the ZigBee wireless network, which obtains reliable, accurate data transmission and data analysis. Its characteristics are at short range, low complexity, low power consumption, low cost, communication distance from standard of 75 meters to hundreds of meters or a few kilometers, and supporting for unlimited expansion. According to the demand of factory aquaculture, and considering the difference between the factory breeding and the standardization pond farming, the structure of wireless sensor network is based on the ZigBee protocol.

In addition, ZigBee network has different network topology of tree, star, etc. The paper is designed with the tree network structure, namely a cluster is constituted with a wireless 


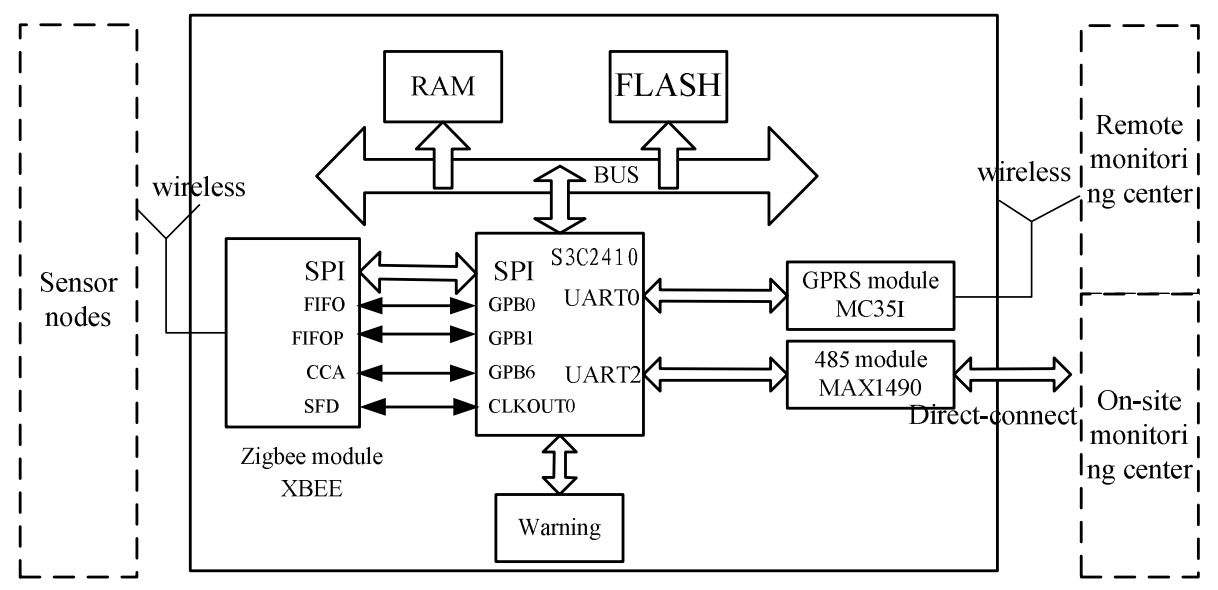

Fig. 1. The structure diagram of the wireless sink node

sink node (primary device), lots of wireless sensor nodes and some wireless control nodes (slave unit), and relay node; then a network is constituted with many clusters [7-8].

On the other hand, the connection of sink node with monitoring centers is in two ways of wired and wireless. Via GPRS network, the sink node could connect with the remote monitoring center. The MC35I GPRS module was designed into the equipment, and its output pins connect directly with the ARM processor's serial port O(UART0). With stable performance and large working temperature range, low power consumption ARM serve as master controller, it can deal with protocol at high speed and a large number of data transmission. Moreover, convenient configuration, long communication distance, wide coverage area, real-time online terminal are all achieved. Based on TCP/IP protocol, it's convenient to manage network with high transmission rate, large data volume, low cost. Based on GPRS technologies, it improves the real-time and reliability of system.

On-site monitoring center can directly connect to sink nodes through cable connection. RS485 communication interface adopt the MAX1490 chip which is a kind of complete photoelectric coupling isolation RS485 data interface chip, simplex work way, maximum transmission baud rate can reach $2.5 \mathrm{Mb} / \mathrm{s}$. Connecting directly with the ARM processor's serial port 2(UART 2), the relevant data of time, coordinate, etc, are read by the ARM processor as broadcasting information. It fully satisfies the needs of different monitoring centers with low cost, strong practical applicability.

\section{Information Fusion}

Because what the monitoring center just pays attention to is the abnormal water quality condition. Only when the environmental conditions are in the abnormal situation may take control measures. If doing Information Fusion at sink node to find abnormal water environment and only sending abnormal water quality data, which can eliminate the redundant information, reduce the amount of data transmission, save energy, prolong the network life effectively, improve the rapidity and correctness of the whole 
monitoring system's response[9-10]. After each collection node power up, do the port and memory initialization first and apply for the ZigBee network. After getting the response of the network, scan each node by the network and then assign the network address that is not used within the given time to the node. Detection of whether the response of sensor is normal. If not, storage the alarm information for preparation to exchange information with the sink node, and at the same time set to 1 at the alarm bite. If it's normal, it can transmit data to sink node at short distance by the ZigBee. Then information fusion can be done at the sink node. The whole process of information fusion is shown in figure 2 .

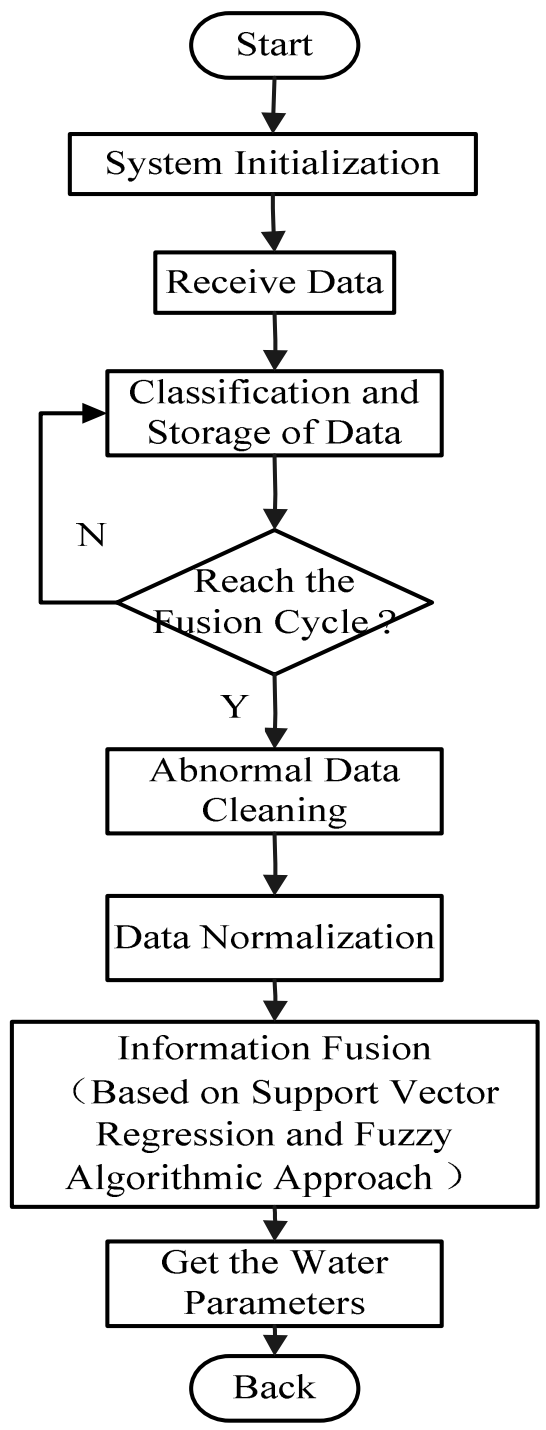

Fig. 2. Information fusion flow chart 
This part of the information fusion is divided into two components, the first part is data fusion pretreatment.

\subsection{Data Fusion Pretreatment}

Data fusion pretreatment includes both abnormal data cleaning and data transformation.

\subsubsection{Abnormal Data Cleaning}

In this section, the main responsibility are abnormal data identification, eliminating noise, correcting the inconsistent data, deleting data redundancy and the completion of detection with sample data that are significant difference with other data in the collected data set. For abnormal data and noise, the principles of management is cleaning and not to participate in the fusion processing. Here we adopt the $3 \delta$ standards based on statistic analysis. Firstly, it's assumption that a set of test data only contain the random error, and carry on calculation processing to get standard deviation, determine a range according to certain probability. It's supposed that exceeding the error range is rather the random error than the gross error, and contains the gross error of data should be rejected. With $3 \delta$ criterion for judging calculation, firstly it's to get the average $x$ of the value $x_{i}$ to replace true value, then residual obtained $v_{i}=x_{i}-x$, getting standard deviation calculated by Bessel formula to replace $\delta$, comparing the value of $3 \mathrm{~S}$ with each residual $v_{i}$, if the residual $v_{i}$ of some suspicious data $x_{i}$ meet next type, the data $x_{i}$ should be gross error and be eliminated.

$$
\left|v_{i}\right|=\left|x_{i}-\bar{x}\right|>3 S
$$

After every obliterating gross error, the rest of data will be recounted S. According to the new numerical that had become smaller $S$, further determining are measured again to judge whether there is gross error until without gross error. Bessel formula is as follow:

$$
S\left(x_{i} k\right)=\sqrt{\frac{1}{n_{i}-1} \sum_{k}\left(x_{i} k-x_{i}\right)^{2}}
$$

\subsubsection{Data Normalization}

In order to eliminate error that is caused by differences in the dimension within different data source, the data from different data source will be normalized.

$$
\hat{x}_{i}=\frac{x_{i}-x_{\min }}{x_{\max }-x_{\min }}
$$

\subsection{Data Fusion}

Completing data pretreatment, the second part of information fusion is data fusion based on Support Vector Regression (SVR) and fuzzy algorithmic approach. 


\subsubsection{Support Vector Regression}

In the factory aquaculture, because of indoor cultivation we can rule out the interference of natural environment, such as wind speed, wind direction, rainfall, etc. What we mainly concern is two factors, the water temperature and dissolved oxygen. Besides, the dissolved oxygen is the most important factor to effect the healthy growth of aquatic products. However, the adjustment of water quality in the breeding relate to what water quality factor are measured accurately and timely. But as circumstances change, there are certain delay itself for dissolve oxygen, and there is also the problem of transmission delay. So, the paper puts forward the information fusion algorithm which gets dissolved oxygen and water temperature as the input parameters of SVR, then the predicted value of dissolved oxygen as the result of information fusion. What the dissolved oxygen of next time is on behalf of water quality conditions make it convenient to control breeding environment timely and accurately [11-12].

The standard SVR algorithm is divided into the linear and nonlinear. The basic idea of the SVR is: it will transform the sample space into another feature space through the nonlinear transformation, and construct regression function in the feature space. The nonlinear transformation could be achieved by defining the appropriate kernel function $K\left(x_{i}, x_{j}\right)$. Besides, $K\left(x_{i}, x_{j}\right)=\varphi\left(x_{i}\right) \cdot \varphi\left(x_{j}\right), \varphi(x)$ is a kind of nonlinear functions.

Given the training data, $\left\{\left(x_{i}, x_{j}\right), i=1,2, \cdots, n\right\}, x_{i}$ is learning samples, and $y_{i}$ is the corresponding target. Define linear insensitive loss function is:

$$
|y-f(x)|_{\varepsilon}=\left\{\begin{array}{cr}
0 & |y-f(x)| \leq \varepsilon \\
|y-f(x)|-\varepsilon & |y-f(x)|>\varepsilon
\end{array}\right.
$$

Namely, if the D-value between the target y and after learning of the structure regression estimation function $\mathrm{f}(\mathrm{x})$ is less than $\mathcal{E}$, the loss is 0 .

With the nonlinear circumstances, the regression estimation function is:

$$
f(x)=\omega^{T} \cdot \phi(x)+b
$$

Looking for a couple of $\omega$ and $b$, which minimize $\frac{1}{2} \omega^{T} \omega$ under the same type (1). Also consider to introduce the relaxation value $\xi_{i}, \xi_{i}^{*}$ when the constraint conditions could not be fulfilled, so the optimization problem is:

$$
\left\{\begin{array}{l}
\operatorname{Min} \frac{1}{2} \omega^{T} \omega+C \sum_{i=1}^{n}\left(\xi_{i}+\xi_{i}^{*}\right) \\
\text { s.t. } y_{i}-\omega \cdot \phi\left(x_{i}\right)-b \leq \varepsilon+\xi_{i} \quad \xi_{i}^{*} \geq 0, i=1,2, \cdots, n \\
\omega \cdot \phi\left(x_{i}\right)+b-y_{i} \leq \varepsilon+\xi_{i}^{*}
\end{array}\right.
$$


Using Lagrange multiplier to solve the constrained optimization problem, so construct the Lagrange function:

$$
L_{P}=\frac{1}{2}\|\omega\|^{2}+C \sum_{i=1}^{n}\left(\xi_{i}+\xi_{i}^{*}\right)-\sum_{i=1}^{n}\left[\varepsilon+\xi_{i}-y_{i}+\omega \cdot \phi\left(x_{i}\right)+b\right]-\sum_{i=1}^{n} \alpha_{i}^{*}\left[\varepsilon+\xi_{i}^{*}+y_{i}+\omega \cdot \phi\left(x_{i}\right)-b\right]-\sum_{i=1}^{n}\left(\beta_{i} \xi_{i}+\beta_{i}^{*} \xi_{i}^{*}\right)
$$

By the optimization theory, make $L_{P}$ to $\omega, b, \xi_{i}, \xi_{i}^{*}$ respectively for asking partial differentia, and make its 0 .

$$
\begin{gathered}
\frac{\partial L}{\partial \omega}=0 \Rightarrow \omega-\sum_{i=0}^{n}\left(\alpha_{i}-\alpha_{i}^{*}\right) \phi\left(x_{i}\right)=0 \\
\frac{\partial L}{\partial b}=0 \Rightarrow \sum_{i=0}^{n}\left(\alpha_{i}-\alpha_{i}^{*}\right)=0 \\
\frac{\partial L}{\partial \xi_{i}}=0 \Rightarrow C-\alpha_{i}-\beta_{i}=0 \\
\frac{\partial L}{\partial \xi_{i}^{*}}=0 \Rightarrow C-\alpha_{i}^{*}-\beta_{i}^{*}=0
\end{gathered}
$$

When applies the type (5) in the type (4), we get the dual optimization problem.

$$
\left\{\begin{array}{c}
\operatorname{Max}\left[-\frac{1}{2} \sum_{i=1}^{n} \sum_{j=1}^{n}\left(\alpha_{i}-\alpha_{i}^{*}\right)\left(\alpha_{j}-\alpha_{j}^{*}\right) K\left(x_{i}, x_{j}\right)-\varepsilon \sum_{i-1}^{n}\left(\alpha_{i}+\alpha_{i}^{*}\right)+\sum_{i=1}^{n} y_{i}\left(\alpha_{i}-\alpha_{i}^{*}\right)\right] \\
\text { s.t. } \sum_{i=1}^{n}\left(\alpha_{i}-\alpha_{i}^{*}\right)=0 \\
0 \leq \alpha_{i} \leq C, 0 \leq \alpha_{i}^{*} \leq C
\end{array}\right.
$$

Support vector (SV) is part of parameters that make $\alpha_{i}-\alpha_{i}^{*} \neq 0$. Through learning and training, we can get the regression estimation function:

$$
f(x)=\sum_{x_{i} e s v}\left(\alpha_{i}-\alpha_{i}^{*}\right) K\left(x_{i}, x\right)+b
$$

We also get:

$$
b=\frac{1}{N_{N S V}}\left\{\sum_{0<\alpha_{j}<C}\left[y_{i}-\sum_{x_{i} e s v}\left(\alpha_{j}-\alpha_{j}^{*}\right) K\left(x_{j}, x_{i}\right)-\varepsilon\right]+\sum_{0<\alpha_{j}<C}\left[y_{i}-\sum_{x_{i} e s v}\left(\alpha_{j}-\alpha_{j}^{*}\right) K\left(x_{j}, x_{i}\right)+\varepsilon\right]\right\}
$$

Besides, the function $K\left(x_{i}, x\right)$, which is known as the Kernel Function. Kernel functions enable dot product to be performed in high-dimensional feature space using low dimensional space data input without knowing the actual transform function $\phi$.All kernel functions must satisfy Mercer' condition that corresponds to the inner product of 
some feature space. The radial basis function (RBF) is commonly used as the kernel for regression:

$$
K\left(\mathrm{x}_{i}, \mathbf{x}\right)=\exp ^{\left\{-\gamma\left|x-x_{i}\right|^{2}\right\}}
$$

After training and learning, we will establish the support vector regression model so as to realize the information fusion of acquisition. Then we will convert the results to practical engineering unit through inverse transformation [13-14].

$$
\bar{x}_{i}=\widehat{x}_{i} *\left(x_{\max }-x_{\min }\right)+x_{\min }
$$

Where $x_{\max }, x_{\min }$ is respectively the maximum and minimum value before normalization in Eq. (3).

\subsubsection{Fuzzy Algorithmic Approach}

Through the analysis of real object, Fuzzy algorithm is developed to process data and construct fuzzy mathematical model. With subordinate relations, the data set was changed into the fuzzy set to determine the membership function. Fuzzy statistics is more than the basis of experience a psychological process. It often works through the psychological measurement, and search the fuzziness of the thing itself [15].

In the paper, we adopt fuzzy algorithmic approach with triangle membership functions to make fuzzy computation for dissolved oxygen that had been calculated via the support vector regression algorithm. Then it's divided into the different water quality abnormal conditions as table 1 :

Table 1. Water quality abnormal state

\begin{tabular}{c|cc}
\hline The DO value & Numerical water quality state & $\begin{array}{c}\text { Water quality state of fuzzy language } \\
\text { description }\end{array}$ \\
\hline$(0,3]$ & 0 & The worst poor \\
$(3,4]$ & 1 & Secondly poor \\
$(4,5]$ & 2 & poor \\
$(5,8]$ & 3 & A bit poor \\
$(8,+\infty)$ & 4 & normal \\
\hline
\end{tabular}

\section{$4 \quad$ Study Site and Implementation}

The practical wireless sink nodes had already been integrated in the water quality monitoring system which was implemented in the factory aquaculture farm of Xin Yongfeng aquaculture Co., LTD, located in Tianjin, China, one of Intensive Aquaculture Research Demonstration Centers of China Agriculture University. The system has stable operated more than one year and has obtained many water quality parameters. 


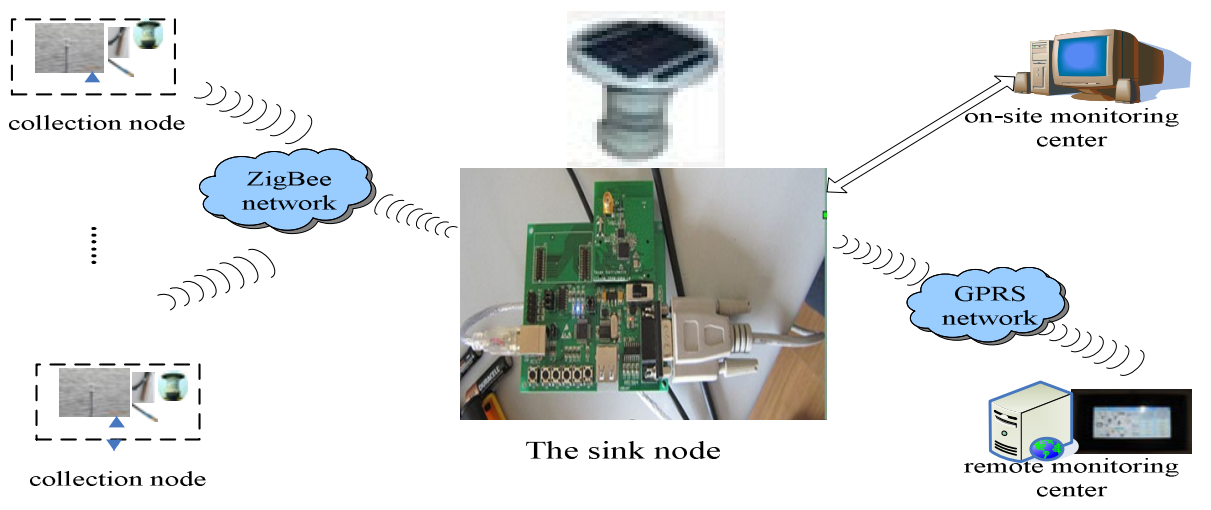

Fig. 3. Water quality monitoring system

The water quality monitoring system is shown in Figure 3. According to the actual demand, the collection nodes include DO sensor and temperature sensor which have the ability of self-calibration, self-compensation and self-diagnosis. Besides, the sensors are solar powered and adopt dormancy strategy to save energy. So the whole working process is that collection nodes collects water temperature and dissolved oxygen, and then sends the data to sink nodes through ZigBee wireless protocol; Secondly, in the sink nodes. making decisions according to information fusion, which converts the collected water quality information into a simple parameter that signified the current state of water quality; On-site monitoring center can directly get the regularly updated data from sink nodes through RS485 interface, then provide the function of data show, data storage and data download; At the same time, the sink nodes can transmit the data to remote monitoring center through GPRS channel, and remote monitoring center can also undertake the works of data receiving, data storage and data download. Besides the monitoring center will take control measures with water environment in abnormal condition.

\section{$5 \quad$ Results and Conclusion}

When being used in the water quality monitoring of factory aquaculture, the water quality monitoring system is stable and can meet the production need. The data which has been adopted in this paper spanned 6 days, from June 8 to June 13, 2011. The sampling interval is 30 minutes, which means 48 sets of data has been collected per day, the total samples is 284 . Figure 4 shows accurate value of water temperature and dissolved oxygen collected by the system.

In this paper, we put forward the information fusion algorithm which gets dissolved oxygen and water temperature as the input parameters of SVR, then the predicted value of dissolved oxygen as the result of information fusion. So we adopt 230sets of data as the training data to train the SVR modes, the last 54 sets of data as testing data to analysis the prediction performance of SVR. After the regression process, we get the optimal function as shown in Eq. (13) that can be used to take prediction. Because the 
sampling interval is 30 minutes, so we predict the dissolved oxygen value of 30 minutes later.

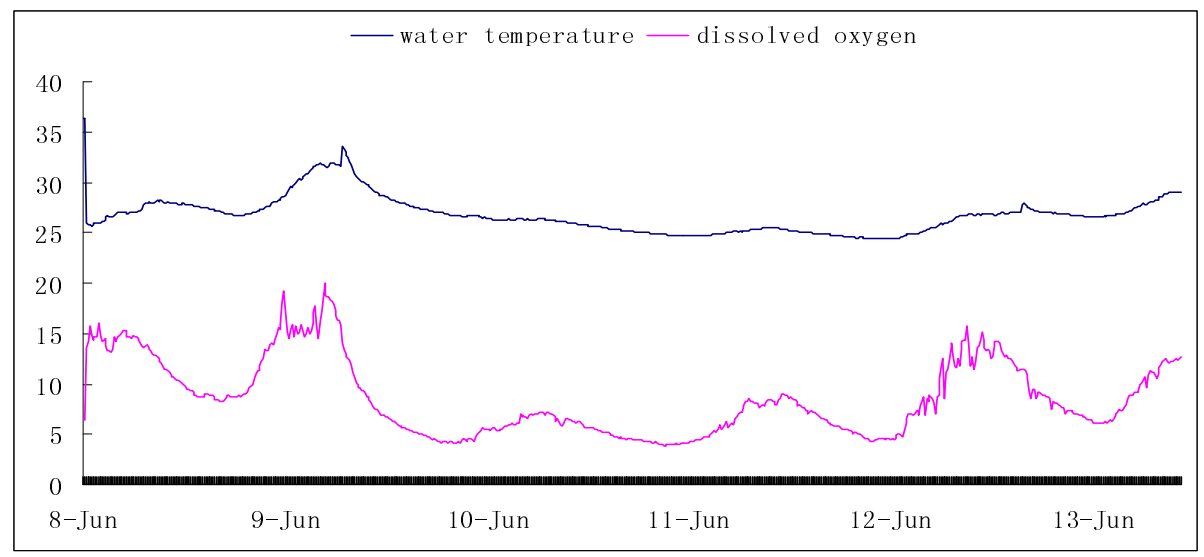

Fig. 4. Accurate value of water temperature and dissolved oxygen

In the experiments, the LIBSVM package had been used for SVM pattern recognition and regression. The package was designed by the Taiwan university professor LinChin-Jen. The specific steps as follows: (1) Format data sets according to the requirements of the LIBSVM package. Put the 230 sets of data that will be used to forecast into a file called "train", the rest of the 54 group into another file called "text". Content with the following format: 8.791 1:8.791 2:27.572, "8.791" on the left side is dissolved oxygen concentration, the rest said two variables influencing DO; (2) Choose the proper kernel function. The radial basis function (RBF) was used as the kernel for regression; (3) Select the optimal parameters with the cross validation method. The principle of the cross validation is dividing the training data into $\mathrm{n}$ parts (here for 10), selection n-1 parts for training every time, leaving one part as test. With MSE as evaluation standards, taking the punishment coefficient $\mathrm{C}, \varepsilon$, kernel function parameter $\gamma$ which are corresponding to the minimum MSE after the traverse all way. And the results can be seen from Table 2; (4) Adopt the optimal parameters to train the whole training set for the support vector machine model and forecast the dissolved oxygen value of 30 minutes later.

At the same time, we can get the Accuracy $=90.7407 \%$ (49/54). It proves the feasibility of the information fusion algorithm which gets dissolved oxygen and water temperature as the input parameters of SVR, then the predicted value of dissolved oxygen as the result of information fusion. Moreover, the prediction precision satisfies our demand, and the sufficient training data guarantee what the test data will forecast results accurately. 
Table 2. The optimal parameters

\begin{tabular}{|c|c|c|}
\hline $\mathrm{C}$ & $\varepsilon \quad(\mathrm{MSE})$ & $\gamma$ \\
\hline 128 & 0.0625 & 0.0078125 \\
\hline
\end{tabular}

\section{Conclusion}

To solve the problems of water quality information collection and satisfy the needs of factory aquaculture farms, this paper designs a sink node using information fusion to integrate into the water quality monitoring system.

The ARM7 architecture of the 32-bit processors was introduced in the sink nodes as the hardware foundation. And depending on the rich peripherals and interface, it can accomplish some full-featured application under the condition of low cost and low power consumption. According to the actual requirements, the multi-mode of communication were adopted as follow: Firstly, the use of GPRS communication module will assist with collection data transmission to the sink nodes; Secondly, the on-site monitoring center could directly get the water quality information that had been processed at the sink nodes through the 485 interface; Furthermore, for the remote monitoring center, the on-site environment parameters and control commands of platform were via the ZigBee wireless network for data transmission. can obtain reliable, accurate data transmission and analytical. It can not only obtain reliable, accurate data transmission and analysis, but also satisfy the different operating requirements of farmers.

What's more, the sink nodes also have the function of information fusion that adopts Support Vector Regression and fuzzy algorithmic approach. According to information fusion, we can make decision to convert the collected water quality information into the simple parameters that could be on behalf of the current state of water quality. The measurement can eliminate redundant information, reduce information transmission, thus save energy effectively and prolong the network life. With information fusion, Water quality abnormal information can be found in real time, so as to improve the reaction validly and accurately of the whole water quality monitoring system.

Besides, the paper puts forward the information fusion algorithm that gets dissolved oxygen and water temperature as the input parameters of SVR, then the predicted value of dissolved oxygen as the result of information fusion. What the dissolved oxygen of next time is on behalf of water quality conditions make it convenient to control breeding environment timely and accurately.

At present, the sink nodes have been applied successfully into the water quality monitoring system, which were implemented at Intensive Aquaculture Research Demonstration Center in Tianjin. So it has a widely application value and market prospect. 
Acknowledgment. This research is financially supported by the Science and Technology Projects of Tianjin Binhai new area: the Integrated Application and Demonstration of Factory Breeding Technology with High Efficiency and Low Carbon, the Twelfth Five-Year-Plant Outline for National Economic and Social Development of the People's Republic of China (2011BAD21B01) and Beijing Natural Science Foundation (4092024). The authors would like to thank the members of Intensive Aquaculture Research Demonstration Center in Tianjin for their hospitality and assistance.

\section{References}

[1] Chatzigiannakis, I., Kinalis, A., Nikoletseasal, S.: Efficient data propagation strategies in wireless sensor networks using a single mobile sink. Computer Communication 31, 896-914 (2008)

[2] Zhang, Y.C., Qian, X., et al.: Field Measurement and Analysis on Diumal Stratification in Taihu Lake. Environmental Science and Management 6, 117-121 (2008)

[3] Alex, H., Kumar, M., Shirazi, B.: Mid Fusion: An adaptive middleware for information fusion in sensor network applications. Information Fusion 9, 332-343 (2008)

[4] Wang, Z., Leung, K.-S., Wang, J.: A genetic algorithm for determining nonadditive set functions in information fusion. Fuzzy Sets and Systems 102, 463-469 (1999)

[5] Bomberger, N.A., Waxman, A.M., Rhodes, B.J., Sheldon, N.A.: A new approach to higher-level information fusion using associative learning in semantic networks of spiking neurons. Information Fusion 8, 227-251 (2007)

[6] Du, Z.G., Xiao, D.Q., et al.: Design of water quality monitoring wireless sensor network system based Oil wireless sensor. Computer Engineering and Design 17, 4568-4570 (2008)

[7] González-Soriano, G., Ortega-Corral, C., González-Vázquez, S., Maeda-Martínez, A.: Remote Web Monitoring and Activation of an Experimental On-off Dissolved Oxygen Concentration Control Model Applied to Aquaculture Tanks. In: 52nd International Symposium ELMAR-2010, September 15-17, pp. 167-171 (2010)

[8] Cheng, C.R., Mao, X.G., et al.: An Online Monitoring System of Water Quality Based on ZigBee. Chinese Joumal of Electron Devices 5, 942-945 (2009)

[9] Jin, N., Ma, R.Z., Lv, Y.F.: A Novel Design of Water Environment Monitoring System Based on WSN. In: ICCDA 2010, China JiLiang University, pp. V2-593-V2-597 (2010)

[10] Yan, M.X.: Short-Term Predicting Model for Dissolved Oxygen of Hyriopsis Cumingii Ponds Based on Elman Neural Network (2011)

[11] Wang, Z., Hao, X.Q., et al.: Remote Water Quality Monitoring System Based ON WSN and GPRS. Instrument Technique and Sensor 1, 48-52 (2010)

[12] Li, C.N., Tan, C.H.H., Kao, S.J., Wang, T.S.: Improvement of remote monitoring on water quality in a subtropical reservoir by incorporating grammatical evolution with parallel genetic algorithms into satellite imagery. Water Research 42, 296-306 (2008) 
[13] Liu, X.Q., Liu, Y., Gong, X.X.: Design of Intelligent Dissolved Oxygen Detecting System Based on CAN Bus and Embedded USB Host. In: 2009 International Conference on Measuring Technology and Mechatronics Automation (2009)

[14] Wei, Y.G.: Prediction of Dissolved Oxygen Content in Aquaculture of Sea Cucumber Using Support Vector Regression. China Agricultural University (2011)

[15] Huang, H.-C.L.L.-T., Lae, L.-F., Chi, Y.-F.: A Remote Automated System for a Case Study of Dissolved Oxygen Monitoring and Control. In: ISIE 2009, pp. 2184-2189. Chienkuo Technology University, Taiwan (2009) 